\title{
Regulation of AP-1 by MAPK Signaling in Metal-Stressed Sea Anemone
}

\author{
Maayan Agron Vera Brekhman David Morgenstern Tamar Lotan \\ Marine Biology Department, The Leon H. Charney School of Marine Sciences University of Haifa, Haifa, \\ Israel
}

\author{
Key Words \\ AP-1 $-\mathrm{MAPK} \cdot \mathrm{GSK} 3-\beta \cdot$ Evolution $・$ Stress $・$ Nematostella vectensis $・$ Cnidaria
}

\begin{abstract}
Background/Aims: AP-1 transcription factor plays a conserved role in the immediate response to stress. Activation of AP-1 members jun and fos is mediated by complex signaling cascades to control cell proliferation and survival. To understand the evolution of this broadlyshared pathway, we studied AP-1 regulation by MAPK signaling in a basal metazoan. Methods: Metal- stressed cnidarian Nematostella vectensis anemones were tested with kinase inhibitors and analyzed for gene expression levels and protein phosphorylation. Results: We show that in cnidarian, AP- 1 is regulated differently than in bilaterian models. ERK2 and ERK5, the main MAPK drivers of AP-1 activation in Bilateria, down-regulated fos 1 and jun 1 transcription in anemones exposed to metal stress, whereas p38 MAPK, triggered transcription of jun 1 but not fos1. Furthermore, our results reveal that GSK3- $\beta$ is the main driver of the immediate stress response in Nematostella. GSK3- $\beta$ triggered transcription of AP-1 and two other stress-related genes, egrl and $h s p 70$. Finally, phylogenetic analysis and protein characterization show that while MAPKs and GSK3- $\beta$ are evolutionarily conserved, Fos and Jun proteins in Nematostella and other cnidarians lack important regulatory and phosphorylation sites found in Bilateria. Conclusion: These findings reveal alternative network interactions of conserved signaling kinases, providing insight into the evolutionary plasticity of immediate stress response mechanisms.

\section{Introduction}

Cells and organisms must respond to changes in environmental conditions in order to survive. Thus, external pressures have driven the evolution of an array of genes and signaling cascades that are rapidly induced upon exposure to extracellular stimuli. Mitogen-activated protein kinases (MAPKs), serine/threonine kinases that catalyze the phosphorylation of transcription factors, protein kinases, and many other substrates including functional proteins, play a central role in mediating cellular responses to extracellular signals $[1,2]$. An 


\section{Cellular Physiology Cell Physiol Biochem 2017;42:952-964

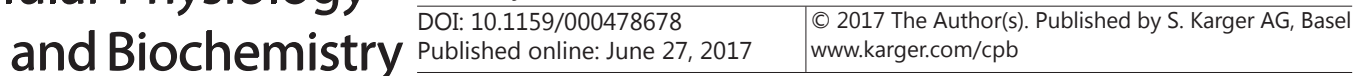 \\ Agron et al.: AP-1 Regulation by MAPKs in Cnidaria}

important target of the MAPKs is the activator protein 1 (AP-1) transcription factor, which is a key regulator of the response to environmental stressors [3-5]. It acts as an environmental biosensor that modulates a wide range of cellular processes such as transcription, proliferation, apoptosis and cancerous transformation [3-5]. AP-1 is a dimeric transcription factor that consists of members of the basic leucine-zipper (bZIP) proteins, mainly of the Fos and the Jun families, which also belong to the immediate-early gene family.

MAPKs regulate AP-1 both at the transcriptional and post-translational levels, affecting its transactivation potential, DNA binding capacity and stability. This network involves complex positive interactions among MAPKs, mainly between the extracellular-signal regulated kinases (ERKs), p38 kinase and c-Jun $\mathrm{N}$-terminal kinase (JNK). Conversely, glycogen synthase kinase 3- $\beta$ (GSK3- $\beta$ ) was shown to act as a negative regulator of AP-1 [6]. Thus, cross-talk between these signaling pathways determines how the environmental signals are transduced into transcriptional regulation of specific sets of genes. To date, AP-1 has been extensively studied in bilaterian model organisms such as mammalians, Planaria, Drosophila and Ascidia [5, 7-12], but little is known about AP-1 regulation and signaling network in basal organisms. Of specific interest is the Cnidaria phylum, which is the eumetazoan group most closely related to bilaterians $[13,14]$. Dating back 700 million years, the Cnidaria consists of more than 10,000 species such as coral, sea anemone, jellyfish and hydra. They have important ecological roles as reef structure builders and as predators and prey in the marine ecosystem. However, the cnidarians and mostly the anthozoans (coral and sea anemones) are in constant decline, endangered by exposure to polluting agents and natural environmental fluctuations $[15,16]$. In particular, heavy metals are major pollutants, because they are passed up the food chain, accumulate in the environment and have prolonged destructive effects that can result in sea anemone disease, coral bleaching and death [17-19]. Nematostella vectensis is a burrowing sea anemone that is widely distributed in costal estuaries [20]. Surprisingly, despite the remarkable evolutionary distance between Cnidaria and vertebrates, the published genome of Nematostella exhibits a high degree of conservation with those of vertebrates [13]. Nematostella tolerates a wide range of anthropogenic and environmental changes and, in recent years, it has become a valuable cnidarian model for molecular ecology and stress regulation studies [21-24]. To understand the molecular pathway by which the metal signal is transduced and induced oxidation stress in Cnidaria, we recently conducted a detailed transcriptomic analysis in Nematostella upon exposure to four metals [25]. Among our findings, we have identified rapid activation of several immediate-early response genes such as the transcription factors early growth response1 (egr1) and fos. Furthermore, fos transcript was shown to be up-regulated by injury in Nematostella [26], during head regeneration in Hydra and during metamorphosis of coral larvae [27-29].

Although cnidarians appear morphologically simple, having only two germ layers, much of their gene sets and signaling pathways are comparable to vertebrates [13]. Therefore, finding how AP-1 is regulated in Cnidaria is important not only for the mechanistic understanding of their immediate defense regulation, but also for revealing ancestral adaptation to environmental changes. In this study, we investigated the regulation of AP-1 genes by MAPKs and GSK3- $\beta$ in Nematostella in response to mercury (Hg) stress. We characterize the Nematostella Fos and Jun as well as MAPK proteins and demonstrate their regulatory and structural divergence from bilaterian. Our findings suggest that in the basal sea anemone, an alternative signaling cascade has evolved using conserved kinase building blocks to regulate AP-1 and other immediate-early genes.

\section{Materials and Methods}

Sea anemone culture

Nematostella anemones were cultured as described previously [25]. 


\section{Cellular Physiology Cell Physiol Biochem 2017;42:952-964 \begin{tabular}{l|l} 
DOI: 10.1159/000478678 & and Biochemistry \\
Published online: June 27, 2017 & $\begin{array}{l}\text { O 2017 The Author(s). Published by S. Karger AG, Basel } \\
\text { www.karger.com/cpb }\end{array}$
\end{tabular} \\ Agron et al.: AP-1 Regulation by MAPKs in Cnidaria}

Metal and kinase inhibitor treatments

Experiments were carried out in triplicates, each containing five sea anemones aged 2-4 months. Anemones were exposed to $\mathrm{HgCl}_{2}$ at a concentration of $200 \mu \mathrm{g} / \mathrm{L}(0.74 \mu \mathrm{M})$ in $10 \mathrm{ml}$ medium for 1,6 and $24 \mathrm{~h}$ and compared to control untreated anemones. The kinase inhibitors were reported to display high sequence specificity and are widely used in different organisms including, to some extent, in Nematostella [26, 3033]; however, their specificity in Nematostella has not yet been established. Kinase inhibitors dissolved in DMSO or only DMSO (control) were added two hours before the introduction of Hg. GSK3- $\beta$ inhibitor alsterpaullone (ALP) (Sigma-Aldrich) was used at $5 \mu \mathrm{M}$ concentration. ERK1/2 inhibitor U0126 (Tocris Bioscience), ERK5 inhibitor XMD 8-92 (Santa Cruz), and p38 inhibitor SB203580 (Cayman chemicals) were used at $10 \mu \mathrm{M}$ concentration.

RNA extraction and $q P C R$

RNA was extracted from Hg-treated and untreated anemones using Tri-reagent (Sigma-Aldrich) and purified as described previously [25]. qPCR was performed using the StepOnePlus Real-Time PCR System (Applied Biosystems) and $\beta$-actin was used as an internal control as described previously [25]. For primers and GenBank accession numbers, (for all online suppl. material, see www.karger.com/doi/10.1159/ 000478678) see Table S1. Results are presented as the means and standard error (SE) of at least three biological replicates. Statistical analysis, comparing treatment to control or between two treatments, was performed using t-test (one sample t-test or independent t-test) by SPSS v.21 software.

Western blot analysis

Experiments were carried out in three biological replicates. Total proteins from each group of three to five treated or control anemones were extracted in RIPA buffer (10 mM Tris (pH 7.0), 2 mM EDTA (pH 8.0), $0.5 \%$ NP-40, $0.05 \%$ deoxycholic acid) freshly supplemented with $1 \mathrm{mM}$ PMSF protease inhibitor and phosphatase inhibitors cocktail containing $10 \mathrm{mM}$ sodium fluoride, $10 \mathrm{mM} \beta$-glycerol phosphate, $10 \mathrm{mM}$ tetrasodium pyrophosphate (Na4P2O7) and $1 \mathrm{mM}$ sodium orthovanadate (Na304V). Samples were boiled and separated on $12 \%$ SDS PAGE and transferred onto nitrocellulose membranes. For immunoblotting, membranes were blocked for 60 min with 5\% dry milk in TBS/0.1\% Tween and probed with antibodies at $4^{\circ} \mathrm{C}$ overnight. Primary antibodies used were: antiphospho-ERK1/2 1:2000 (\#4370, Cell Signaling Technology), anti-ERK1/2 1:30000 (M5670, Sigma-Aldrich), antiphospho-p38 1:1000 (\#9211, Cell Signaling Technology), anti-Actin 1:1000 (sc-1615, Santa Cruz). Secondary antibodies were bovine antigoat-HRP 1:10000 and goat anti-rabbit-HRP 1:10000 (Jackson Immunoresearch). Reaction was performed in ECL solution (Advansta Corporation) and bands were visualized using ImageQuant LAS 4000. Western blot antibodies were stripped in $0.1 \mathrm{M}$ glycine ( $\mathrm{pH} 2.5)$ and incubated with an antibody against actin or ERK1/2.

\section{Phylogenetic Analysis}

Multiple sequence alignment based on protein sequences was performed with MAFFT alignment software (version 7.3) [34], using either single domain (E-INS-i) or multiple domain (L-INS-i) alignment strategies, followed by alignment assessment using DAMBE (version 5) [35]. Phylogenetic analysis was performed using MrBayes software [36] by running two independent chains of four threads each, using mixed amino acid model with gamma distribution with or without a proportion of invariant sites. Analyses were run for 5 million generations, sampling each 100 generations, for standard deviation of $<0.01$. Tree quality, i.e. coalescence and effective sample sizes, was assessed using Tracer 1.6 [37] (http://beast.bio. ed.ac.uk/Tracer). The first 20000 trees were burned, and the choice between gamma and invgamma distribution models was made based on the Tracer analysis. FigTree software (version 1.4.2) was used for visualization of the trees. GenBank accession numbers of the analyzed proteins are provided in (see supplementary material) Table S2.

\section{Results}

Nematostella AP-1 subunits are up-regulated by metal stress and contain partially conserved phosphorylation sites

To study AP-1 activation by metal signal transduction, we initially searched for AP-1 homolog genes in the Nematostella genome. Two homologs of the Fos family: fos 1 and fos 2 
and three homologs of the Jun family: jun1, jun2 and jun3 were found. qPCR analysis showed that only fos 1 and jun 1 transcripts are highly up-regulated in response to $\mathrm{Hg}$. Elevation in both gene transcripts appeared as early as $1 \mathrm{~h}$ after exposure and transcript levels decreased after $6 \mathrm{~h}$ and $24 \mathrm{~h}$ (Fig. 1). Protein structure analysis showed that Jun proteins were relatively conserved in Nematostella, containing known phosphorylation sites and trans-activating domains of Bilateria (Fig. 2), with the exception of GSK3- $\beta$ phosphorylation sites. Specifically, Jun1 contained only one of three known human GSK3- $\beta$ regulatory sites; however, the Ser 243 (human c-Jun index), which is essential for phosphorylation of all three GSK3- $\beta$ sites, was missing from all tested cnidarians [4, 38-40] (Fig. 2). Nematostella Fos proteins were found to be shorter and were missing the Bilateria regulatory C-terminal end, including two trans-activating domains known as homology box 1 and 2 (HOB1 and HOB2) and a docking site for ERK (DEF domain) (Fig. 2) [4]. Analysis of Fos in other cnidarian species representing three different classes, namely Aurelia aurita (Scyphozoa), Hydra vulgaris (Hydrozoa) and Acropora digitifera (Anthozoa), revealed similarly shorter sequences (Fig. 2).

To examine the evolutionary relationships between Jun and Fos proteins in Nematostella and in known bilaterians, we constructed a phylogenetic tree. The results reveal the monophyly of the bilaterian and cnidarian sequences (Fig. 3). This monophyly suggests that the split between Bilateria and Cnidaria occurred prior to the neofunctionalization of jun and fos genes as currently evident in Bilateria, especially regarding the acquisition of domains in Bilateria Fos proteins that are absent in Cnidaria. However, we observed that the Jun and Fos subfamilies within Cnidaria display polyphyly, suggesting that they diverged after the main clades of Cnidaria had split.

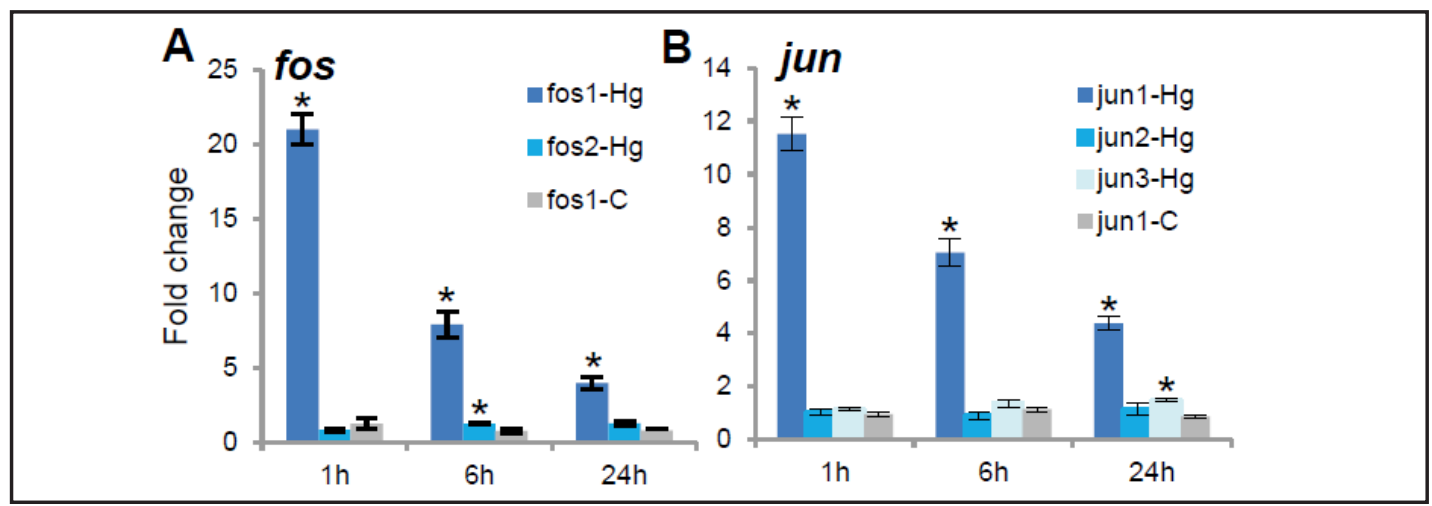

Fig. 1. Time course of AP-1 subunit expression. qPCR analysis of Hg-treated and untreated anemones for the expression of fos (A) and jun (B) AP-1 subunits. Results $(n \geq 3)$ are presented as the average fold change \pm SE relative to control anemones. Asterisks indicate statistically significant differences $(P<0.05)$.

Fig. 2. Comparison between human c-Jun and c-Fos and Nematostella vectensis, Acropora digitifera (coral), Aurelia aurita (jellyfish) and Hydra vulgaris Jun and Fos protein homologs. The main domains of the AP-1 proteins are demonstrated including: basic region for DNA interaction, leuzin zipper for protein dimerization, HOB1 and 2 for transactivation, DEF for docking site for ERK and Delta for docking site for JNK. The ERK phosphorilation sites in c-Fos and the GSK3- $\beta$ phosphorilation sites in c-Jun and their putatives sites in the cnidarians are marked.

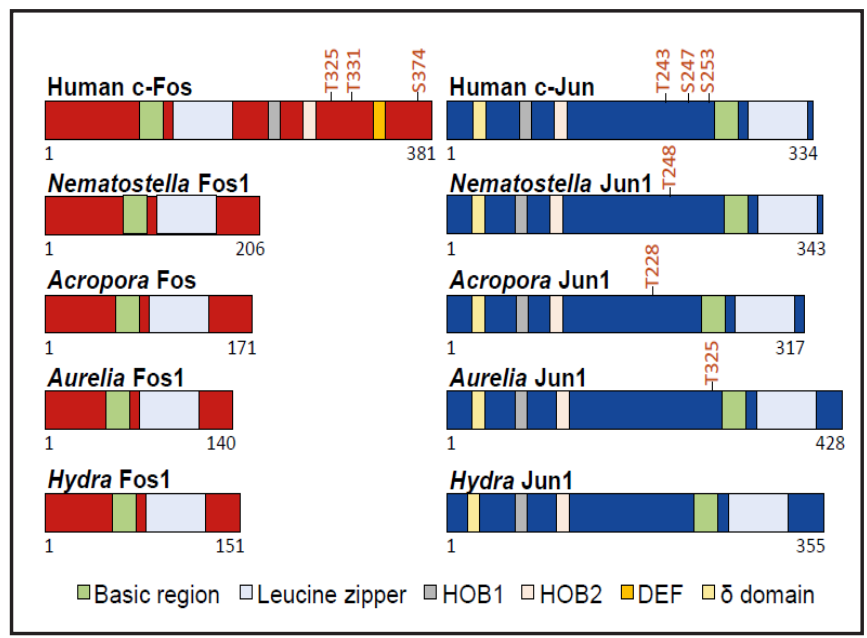


Fig. 3. Rooted Bayesian phylogenetic tree of Fos and Jun AP-1 proteins. The following species are shown: Bilateria: Homo sapiens (Hs), Rattus norvegicus (Rn), Gallus gallus (Gg), Danio rerio (Dr), Ciona intestinalis (Ci) and Drosophila melanogaster (Dm); Cnidaria: Acropora digitifera (Ad), Hydra vulgaris (Hv), Aurelia aurita (Aa) and Nematostella vectensis (Nv). The sponge Amphimedon queenslandica (Aq) was used as an out-group. Scale bar represents amino acid substitutions per site. For the full proteins list, see supplementary material, Table S2.

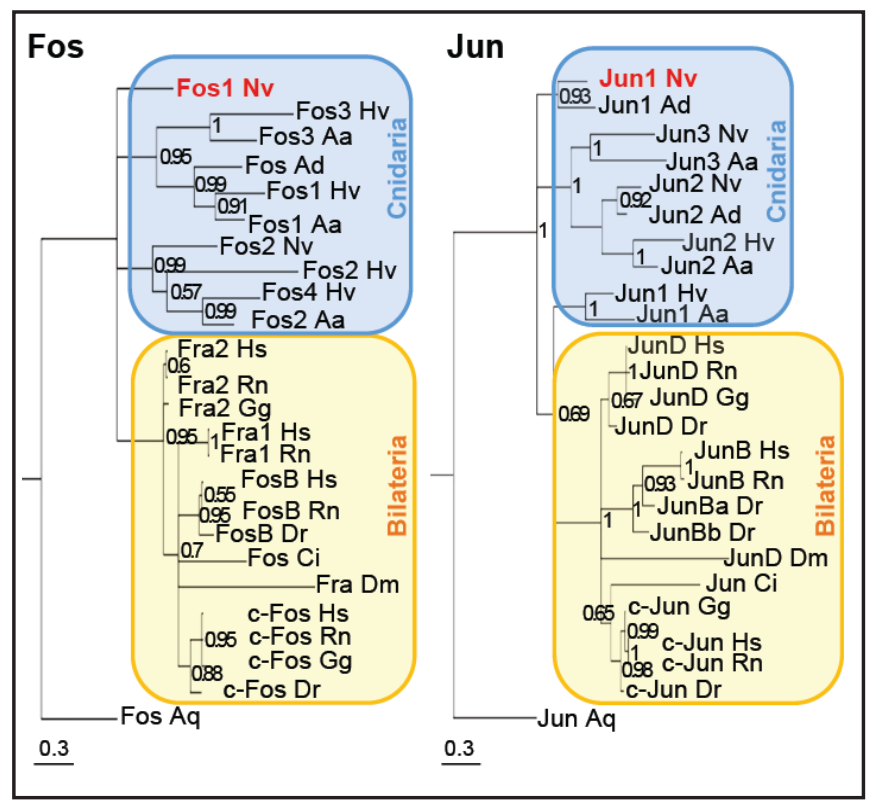

Altogether, these results suggest that cnidarian AP-1 proteins lack part of the most conserved regulatory domains of Bilateria, thus raising the question of how Fos and Jun are regulated in cnidarians. Given the observed up-regulation of fos 1 and jun 1 during metal stress, we continued our analysis of AP-1 regulation in Nematostella using these transcripts.

\section{p38 up-regulates jun1 but not fos1}

In Bilateria, p38 signaling pathway is known to be stimulated by external or cellular stress and to induce AP-1 transcription [4]. Therefore, we asked if this MAPK has a similar effect in Nematostella. Since Nematostella MAPKs have not yet been fully characterized, we first searched for p38 homologs. In human, p38 MAPK family consists of four proteins, namely $\mathrm{p} 38 \alpha / \beta / \gamma / \delta$. Yet, analysis showed that Nematostella has only one p38 homolog most resembling p38 $\alpha$ (67\% protein identity with human), which is the main p38 MAPK in mammalians where it is expressed ubiquitously [41]. In addition, Nematostella p38 possessed the conserved double-phosphorylation TGY motif, which is required for its activation [42, 43].

Next, we asked if the p38 signaling cascade is activated when the anemones are exposed to $\mathrm{Hg}$ stress. Immunoblotting using a phospo-p38 cross-reactivity antibody revealed that Nematostella p38 is indeed phosphorylated in response to metal stress, whereas in untreated control anemone p38 is not phosphorylated (Fig. 4A). To determine whether p38 takes part in AP-1 activation, the anemones were exposed to $\mathrm{Hg}$ in the presence or absence of the p38-specific kinase inhibitor SB203580, which blocks p38 activity by preventing phosphorylation of its downstream targets [44]. qPCR results showed that in the presence of $\mathrm{Hg}$, fos 1 transcript levels were not affected by inhibition of p38 activity (Fig. 4B). In contrast, inhibiting p38 kinase activity resulted in a significant reduction of jun1 transcript levels $1 \mathrm{~h}$ and $6 \mathrm{~h}$ after Hg treatment (Fig. 4C). In untreated control anemones, SB203580 had no effect on AP-1 transcription. Although the downstream target phosphorylated by p38 is still unknown, these data demonstrate that the metal stress triggers p38 phosphorylation that, in turn, promotes transcriptional activation of jun1, but not fos1. Therefore, we suggest that in Nematostella p38 signaling cascade regulates AP-1 component jun1.

\section{ERK signaling pathways down-regulate AP-1 transcription}

Because ERK1/2 and ERK5 are among the most established drivers of fos activation in Bilateria $[4,45,46]$, we proceeded to examine the evolutionary conservation of their role. Protein analysis revealed that ERK2 and ERK5 Nematostella homologs possess the conserved 
Fig. 4. The effect of p38 on AP-1 expression. (A) Anemones were exposed to $\mathrm{Hg}$ for $1 \mathrm{~h}, 6 \mathrm{~h}$ and $24 \mathrm{~h}$ and protein extracts were subjected to immunoblot analysis using antibodies against phospho-p38 and $\beta$-actin. Data are representative of three independent experiments. (B, C) qPCR analysis of fos1 (B) and jun1 (C) in anemones that were exposed to $\mathrm{Hg}$ in the presence or absence of $10 \mu \mathrm{M}$ of p38 inhibitor SB203580; at $1 \mathrm{~h}$, a control anemone treated only with SB203580 is shown. Results $(n \geq 3)$ are presented as the average fold change \pm SE. Asterisks indicate statistically significant differences from Hg-treated anemones $(P<0.05)$.

Fig. 5. The effects of ERK2 and ERK5 on AP-1. (A) Anemones were exposed to $\mathrm{Hg}$ for $1 \mathrm{~h}, 6 \mathrm{~h}$ and $24 \mathrm{~h}$ in the presence or absence of $10 \mu \mathrm{M}$ ERK2 inhibitor U0126. Protein extracts were subjected to immunoblot analysis using antibodies to phospho-ERK1/2, ERK1/2 and $\beta$-actin. Data are representative of three independent experiments. (B) Immunoblot of anemone extracts after 1 h exposure to $\mathrm{Hg}$ with or without 10 $\mu \mathrm{M}$ ERK5 inhibitor XMD 8-92 using antibodies to phospho-ERK1/2, ERK1/2 and $\beta$-actin. Data are representative of three independent experiments. Note that ERK2 phosphorylation is not affected by XMD 8-92. (C, D) qPCR analysis of fos 1 (C) and jun1 (D) in Hg-treated anemones in the presence or absence of ERK1/2 and ERK5 inhibitors, or with a combination of the two inhibitors. DMSO- and inhibitor-treated anemones are shown as controls. Results $(n \geq 3)$ are presented as the average fold change \pm SE. Asterisks indicate statistically significant differences from Hg-treated anemone or between inhibitor-treated anemone (without $\mathrm{Hg}$ ) and DMSO control anemones $(P<0.05)$.
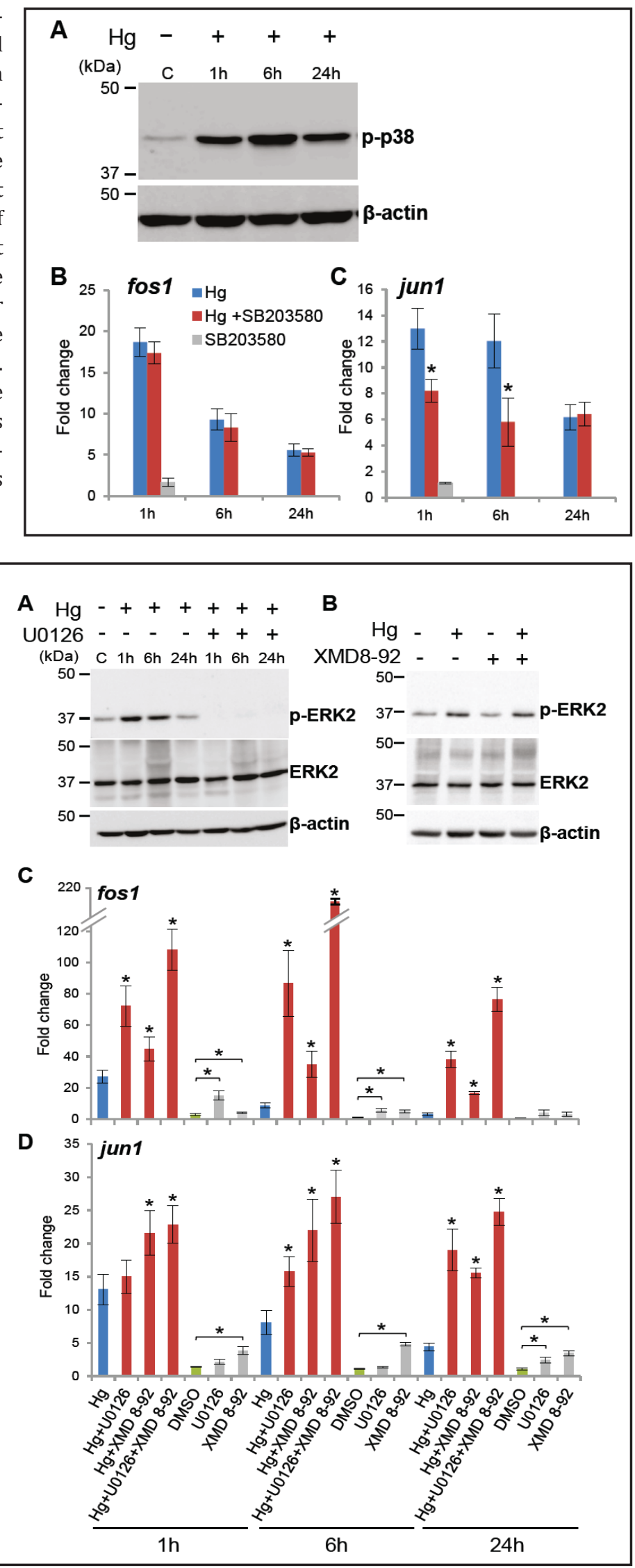


\section{Cellular Physiology Cell Physiol Biochem 2017;42:952-964 \begin{tabular}{l|l} 
DOI: 10.1159/000478678 & Ond Biochemistry \\
Published online: June 27, 2017 & $\begin{array}{l}\text { 2017 The Author(s). Published by S. Karger AG, Basel } \\
\text { www.karger.com/cpb }\end{array}$
\end{tabular} Agron et al.: AP-1 Regulation by MAPKs in Cnidaria}

TEY phosphorylation activation motif and the common putative docking domains of MEK [47], demonstrating structural similarity to Bilateria. However, we found only one homolog of ERK1/2 in Nematostella, most resembling ERK2 (80.7\% protein identity). To directly test the role of ERK2 and ERK5 signaling cascades in Hg-treated anemone we used the MEK1/2-specific inhibitor U0126, which was shown to inhibit ERK1/2 phosphorylation and activation in Nematostella [30, 31], the ERK5 inhibitor XMD 8-92 [48], or a combination of the two. Results showed that Hg induces Nematostella ERK2 phosphorylation and that U0126 completely inhibits ERK2 phosphorylation (Fig. 5A). Although we could not demonstrate ERK5 protein phosphorylation due to the lack of specific phospo-ERK5 antibodies, ERK5 inhibitor did not inhibit ERK2 phosphorylation in the presence of Hg (Fig. 5B). Moreover, ERK2 and 5 inhibitors did not prevent p38 phosphorylation and p38 inhibitor did not prevent ERK2 phosphorylation, suggesting that these two pathways are independent of one another (see supplementary material, Fig. S1).

Next, we examined the levels of AP-1 genes in the presence of the inhibitors. Surprisingly, qPCR results showed more elevated transcription of fos 1 and jun1 in the presence of either ERK1/2 or ERK5 inhibitors in Hg-treated anemone compared to anemone treated only with $\mathrm{Hg}$ (Fig. $5 C$ and $D$ ). fos 1 transcript level increased rapidly within $1 \mathrm{~h}$ in the presence of U0126 and to a lesser extent in the presence of XMD 8-92. jun1 transcript level increased rapidly in the presence of XMD 8-92, whereas an effect of U0126 was demonstrated only after $6 \mathrm{~h}$. However, when both inhibitors were used, an additive effect was observed after $1 \mathrm{~h}$ and after $6 \mathrm{~h}$, a synergistic effect was demonstrated by further elevation in fos 1 transcript level. Interestingly, without the addition of $\mathrm{Hg}$, the two inhibitors also caused substantial increase in fos 1 and jun1 transcripts as compared to DMSO-treated control anemones; specifically, U0126 had a greater effect on fos1 and XMD 8-92 on jun1.

Together, these results indicate that the ERK5 inhibitor has no cross-reaction with ERK2 and that although Hg triggers ERK2 phosphorylation in Nematostella, both ERK2 and ERK5 signaling pathways induce transcriptional repression of AP-1, which is the opposite of their function in Bilateria.

Fig. 6. Phylogenetic analysis of MAPKs. (A) Unrooted protein Bayesian inference phylogenetic tree of MAPK, which includes vertebrates (black): Danio rerio (Dr), Rattus norvegicus (Rn) and Homo sapiens (Hs), cnidarians (blue): Acropora digitifera (Ad), Hydra vulgaris (Hv), Aurelia aurita (Aa) and Nematostella vectensis (Nv), Placozoa (orange) Trichoplax adhaerens (Tr), Porifera (red) Amphimedon queenslandica(Aq), and Ctenophora (green) Mnemiopsis leidyi (Ml). Scale bar represents amino acid substitutions per site. (B) Table representing the number of genes per MAPK family in the

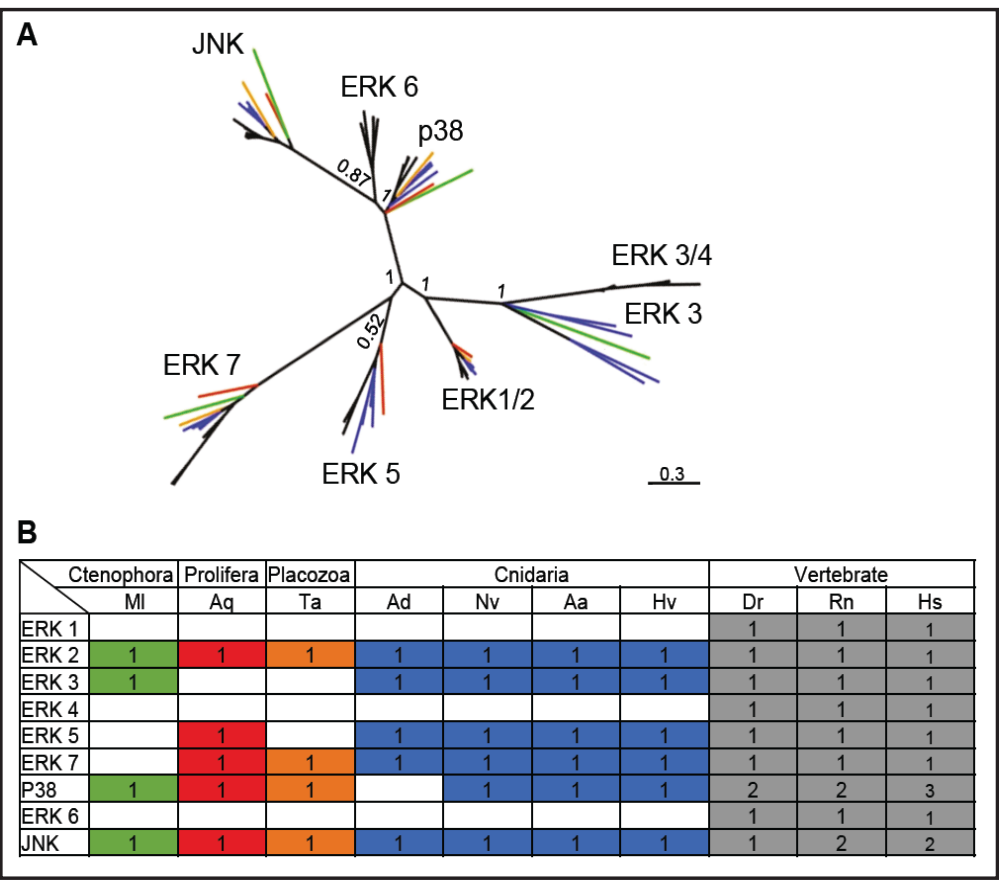
different groups, which are color-coded as in the phylogenetic tree. For the full protein list, see supplementary material, Table S2. 
Phylogenetic analysis of MAPKs

The above results suggested that the MAPK cascade operates differently in Cnidaria. In order to reconstruct the evolution of the MAPK family and to examine MAPK protein conservation, we performed a phylogenetic analysis. We used a Bayesian inference tree based on protein sequences from Bilateria, Nematostella and representatives of three other cnidarians classes, as well as three basal groups including Porifera, Placozoa and Ctenophora (Fig. 6). Analysis revealed seven well-supported clades of the MAPK subfamilies ERKs, p38 and JNK, which were divided according to different functional families of MAPK proteins and not by phyletic association to Bilateria or Cnidaria. Interestingly, all Cnidaria species examined as well as the three other basal organisms exhibited only ERK2, one subunit of p38 and one type of JNK (Fig 6B). In addition, ERK4 and ERK6 were absent from Cnidaria and the three basal organisms indicating that these MAPKs evolved after the Cnidaria-Bilateria split. These results strengthen the structural and functional conservation of the cnidarian MAPKs and their ancient origin.

\section{GSK3- $\beta$ signaling pathways activate AP-1 transcription independently of ERKs}

Searching for a positive transcriptional regulator of AP-1 we tested GSK3- $\beta$, which is known to interact with the Ras/Raf/ERK pathway [49]. Nematostella GSK3- $\beta$ contained the conserved phosphorylation sites known in Bilateria $[6,50]$ and its inhibitor alsterpaullone (ALP) is commonly used in Cnidaria, including in Nematostella [32, 33]. Using ALP in the presence of $\mathrm{Hg}$ caused a reduction in fos 1 and jun1 transcript levels after $1 \mathrm{~h}$, suggesting that GSK3- $\beta$ is an activator of AP-1 transcription in Nematostella (Fig. 7A and B). In control

Fig. 7. The effect of GSK3- $\beta$ on AP-1. (A, B) qPCR analysis of fos1 (A) and jun1 (B) in anemones that were exposed to $\mathrm{Hg}$ for 1,6 , and $24 \mathrm{~h}$ in the presence or absence of $5 \mu \mathrm{M}$ of GSK3- $\beta$ inhibitor ALP and in control ALP-treated anemone. (C) Immunoblot of anemone extracts after $1 \mathrm{~h}$ exposure to $\mathrm{Hg}$ with or without GSK3- $\beta$ inhibitor ALP using antibodies to phospho-ERK1/2, ERK1/2 and $\beta$-actin. Data are representative of three independent experiments. (D-G) qPCR analysis of fos1 (D), jun1 (E), hsp70 (F) and egr1 (G) after 1h in Hg-treated or untreated anemones in the presence or absence of GSK3- $\beta$, ERK1/ 2 and ERK5 inhibitors or in combination of the inhibitors. Results $(n \geq 3)$ are presented as the average fold change \pm SE. Asterisks indicate statistically significant differences of Hg-inhibitor-treated anemone to Hg-treated anemones, or between ERKs inhibitors-treated anemones in the presence or absence of GSK3- $\beta$ inhibitor $(P<0.05)$.

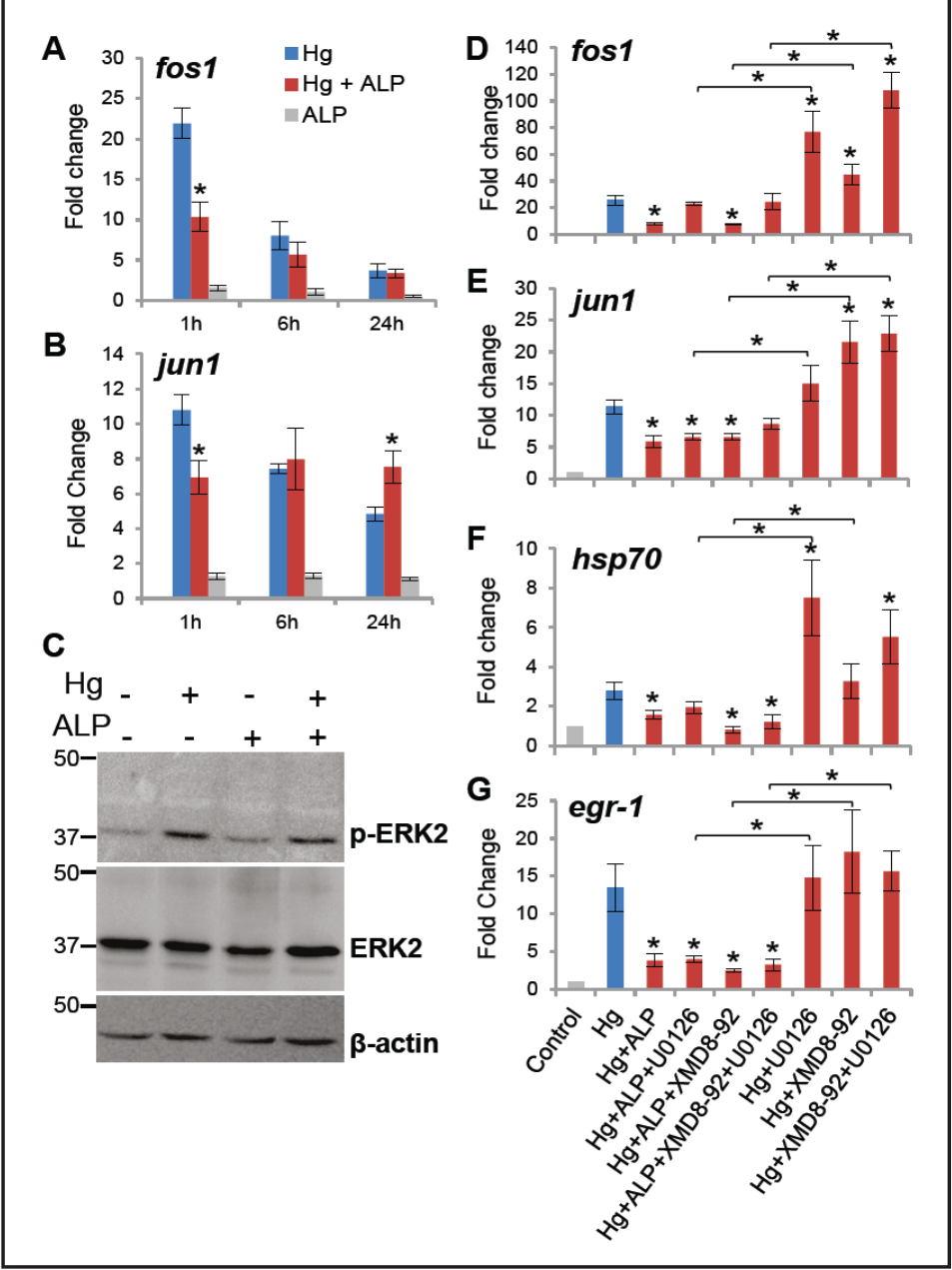




\section{Cellular Physiology Cell Physiol Biochem 2017;42:952-964

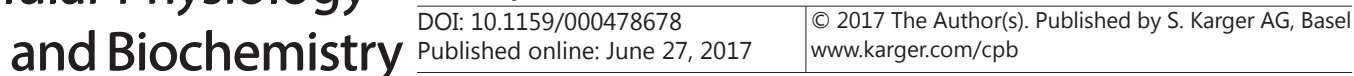 \\ Agron et al.: AP-1 Regulation by MAPKs in Cnidaria}

untreated anemones, ALP had no effect on AP-1 transcription. We also demonstrate that GSK3- $\beta$ has no effect on ERK2 activation, as ALP did not affect ERK2 phosphorylation (Fig. $7 C$ ). Additionally, ALP did not affect p38 phosphorylation (see supplementary material, Fig. S1). These results indicate that GSK3- $\beta$ signaling cascade has a positive activating function on AP-1 transcription in Nematostella, whereas in Bilateria, GSK3- $\beta$ is a negative regulator of c-Jun in unstimulated cells and its inhibitory affect is released upon stress stimulation.

Next, to determine whether GSK3- $\beta$ operates under ERK cascade, we exposed anemones to Hg combined with ALP, U0126 or XMD 8-92, or with different combinations of the three inhibitors. Comparison between treatments with ERK inhibitors with or without ALP (Fig. $7 D$ and $E$ ) clearly showed that the addition of ALP diminished the increase of both fos 1 and jun1 caused by ERK inhibitors; however, in the presence of U0126 the levels of fos 1 were still higher than upon ALP inhibition alone. Together, these results suggest that GSK3- $\beta$ triggers transcription of the AP-1 genes and that it operates through a parallel pathway to ERK2.

\section{Regulation of stress-related genes by GSK3- $\beta$ and ERKS}

To discover if Hg-exposure regulates additional stress-related genes in addition to AP1 we tested the transcription factor egr1, which belong to the immediate-early genes family, and the heat shock protein 70 (hsp70), a known stress response gene. qPCR analysis of early expression $(1 \mathrm{~h})$ of egr 1 and $h s p 70$ showed that both transcripts were up-regulated in the presence of $\mathrm{Hg}$. The addition of ERKs or GSK3- $\beta$ inhibitors showed that upon metal exposure, both genes were down-regulated by GSK3- $\beta$ inhibition (Fig. $7 F$ and $G$ ). However, ERK inhibitors had different effects on the two genes. Whereas egr1 transcript levels were not affected by these inhibitors, $h s p 70$ was regulated more similarly to AP-1 genes with a relatively high transcript expression in the presence of U0126. We conclude that GSK3- $\beta$ plays a major role in mediating metal stress response by rapidly activating members of the immediate-early family and other stress-related genes.

\section{Discussion}

AP-1 is an important transcription factor controlling cell proliferation, survival and death $[4,5,10]$. In Bilateria, AP-1 activity requires protein dimer interactions, as well as specific DNA binding sites, transactivation domains and regulatory phosphorylation sites $[4,9]$. We show that in Nematostella and other organisms that represent the three major Cnidaria classes Anthozoa, Hydrozoa and Scyphozoa, and in in Porifera, Fos proteins are relatively short and lack the HOB transactivation domains, ERK docking site and conserved c-Fos regulatory phosphorylation sites. Furthermore, Fos homolog is completely missing from the Placozoa genome [51]. Yet, the interaction between Fos and Jun proteins was suggested to have existed already in the Eumetazoan ancestor [52, 53]. AP-1 induction by ERK involves both transcriptional and post-translational activation. Yet, ERK was found to affect fos 1 transcription despite the lack of Fos regulatory $\mathrm{C}$ terminal end. This may suggest that in Cnidaria, Fos has additional ERK activation sites yet to be discovered, or that AP-1 transcriptional regulation relies on activation of AP-1 complex by the conserved phosphorylation and transactivation domains of Jun proteins [39]. Cnidaria has also evolved Fos and Jun protein sub-families, which may provide additional dimerization combinations and possibly complex gene regulation $[51,53]$; however, their specific roles remain to be determined.

In Bilateria, AP-1 is regulated by MAPK signaling cascade that is triggered by external stimuli [4]. In Cnidaria, AP-1 was suggested to mediate stress responses such as upon elevation in seawater temperature and injury as well as exposure to heavy metals, which we previously showed to affect fos transcript $[25,26,28,54]$. Here, we show the involvement of MAPKs in the regulation of AP-1 transcripts fos 1 and jun 1 in the presence of Hg. Our phylogenetic analysis shows that the seven subclasses of the MAPK family in Cnidaria are highly conserved, though ERK1 was found to be missing from Nematostella and all other 


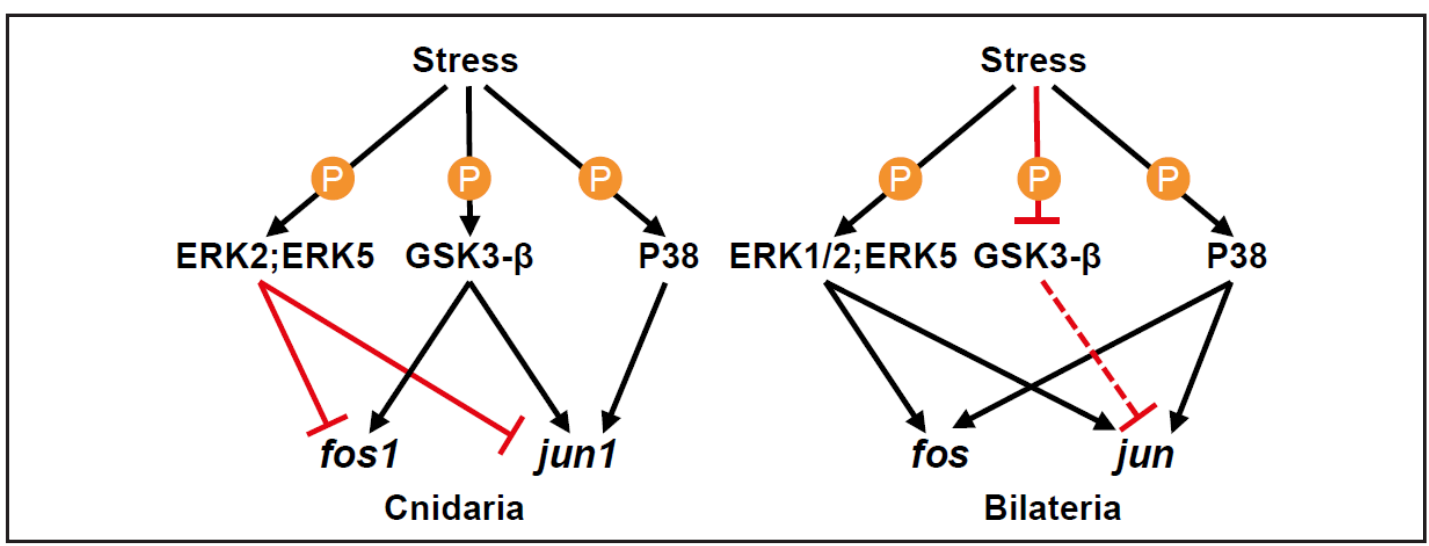

Fig. 8. Proposed model for AP-1 regulation under stress in Cnidaria in comparison to Bilateria. The immediate response to external stress is mediated by AP-1, which subsequently modifies processes such as cell proliferation and survival. In both Cnidaria and Bilateria, metal stress affects p38, ERK and GSK3- $\beta$ signaling cascades; yet, the network wiring differs greatly. In Cnidaria, GSK3- $\beta$ serves as a positive regulator of both AP-1 subunits fos 1 and jun1, whereas p38 triggers up-regulation of only jun1. ERK signaling cascades serve as a negative feedback control mechanism, causing down-regulation of fos 1 and jun 1 transcription. In comparison, in Bilateria stress triggers p38 and ERK signaling cascades, which in return activate transcription of both jun and fos. However, GSK3- $\beta$ signaling is inhibited, releasing Jun protein from the constitutive inhibitory effect (dash line) of this pathway during rest.

examined cnidarians. In addition, ERK1 was also missing from the other tested basal organisms Placozoa, Porifera and Ctenophore. Therefore, it is likely that ERK2 is the ancient form and ERK1 evolved after the Cnidaria-Bilateria split by gene duplication. Single-celled organisms such as yeast and amoeba slime molds also have ERK homologs, yet they consist a separate group [42]. In mammalians, the functions of ERK1 and ERK2 were shown to be similar, though distinctive functions at specific cells or life stages may exist [2].

Our findings demonstrate that Hg triggers MAPK signaling cascade, yet the downstream cascade is different than what is known in Bilateria (Fig. 8). Upon Hg exposure, p38 signaling cascade triggered the transcription of jun 1 but not fos 1 . This is a surprising result, because p38 was shown to up-regulate c-fos in Bilateria following exposure to heavy metals and other stressors $[4,5,55]$.

ERK2 and ERK5 are important activators of AP-1 in Bilateria. We show that Nematostella ERKs contain conserved phosphorylation sites and MEK docking sites as in Bilateria [47] and that Nematostella ERK2 has high similarity to human ERK2. However, although we found that ERK2 was phosphorylated in the presence of $\mathrm{Hg}$, inhibition of ERK2 activation and also of ERK5 induced rapid increase in fos 1 and jun1 transcription. In a recent wound healing study in Nematostella, it was also shown that U0126 triggered fos 1 transcription upon injury [26]. These results indicate that ERK2 and ERK5 down-regulate AP-1 transcription during stress. We suggest that once ERK is phosphorylated, the signaling cascade acts as a negative feedback control mechanism decreasing fos 1 and jun1 transcription to basal levels (Fig. 8). In line with these results, we also found that in non-treated anemones, ERKs were involved in a constitutive inhibition of AP-1. Since Fos1 has no docking site for ERK, this effect may be mediated by an unknown protein either by inhibition of a strong activator of fos 1 or by activation of fos 1 inhibitor.

Another key regulator of AP-1 is GSK3- $\beta$, a multifunctional kinase that takes part in various cellular processes $[6,56]$. In mammals, GSK3- $\beta$ is a negative regulator of AP-1 acting through constitutive inhibitory phosphorylation of c-Jun, which reduces its DNA binding capability and stability $[49,57]$. GSK3- $\beta$ may also affect AP-1 transcription by mutual inhibition with ERK [49]; however in stress conditions GSK3- $\beta$ is inactivated in mammals promoting AP-1 transcription. Our results reveal a different effect, as inhibition of 
GSK3- $\beta$ reduced AP-1 transcription in Nematostella treated with metal and had no effect in untreated anemones, suggesting that GSK3- $\beta$ activates AP-1. As Nematostella jun 1 has only one of the three known GSK3- $\beta$ deactivating phosphorylation sites, the inhibitory potential of GSK3- $\beta$ under normal conditions might be impaired. Using combinations of GSK3- $\beta$ and ERK inhibitors caused a decrease in AP-1 transcription, suggesting that GSK3- $\beta$ is the main regulator of AP-1 in Nematostella, operating in a signaling cascade parallel to ERK proteins. Consistently, we demonstrate that egr1 and $h s p 70$, two other stress related genes, were also rapidly up-regulated by GSK3- $\beta$ when exposed to $\mathrm{Hg}$, similarly to AP-1 but very differently than in tested Bilateria [58].

In conclusion, we identify an ancient defense mechanism consisting of the earlyimmediate genes fos 1 , jun 1, egr1 and the stress gene $h s p 70$, which are rapidly up-regulated during metal stress in a basal metazoan. Our results indicate that while MAPKs and GSK3- $\beta$ are important and conserved regulators of environmental stress, their network interaction has dramatically changed during evolution. These findings shed light on the evolutionary plasticity of the molecular networks mediating immediate stress response and may contribute to the study of yet unknown Bilateria stress network interactions.

\section{Acknowledgments}

We thank the Bioinformatics Service Unit at University of Haifa for their assistance with the phylogenetic analysis. The work was supported by the Israeli Ministry of Science and Technology (MOST). The funder had no role in study design, data collection and interpretation, or the decision to submit the work for publication

\section{Disclosure Statement}

The authors state that they have no conflict of interest.

\section{References}

1 Plotnikov A, Zehorai E, Procaccia S, Seger R: The MAPK cascades: Signaling components, nuclear roles and mechanisms of nuclear translocation. Biochim Biophys Acta Mol Cell Res 2011;1813:1619-1633.

2 Roskoski JR: ERK1/2 MAP kinases: Structure, function, and regulation. Pharmacol Res 2012;66:105-143.

-3 Karin M, Liu Z-g, Zandi E: AP-1 function and regulation. Curr Opin Cell Biol 1997;9:240-246.

- Eferl R, Wagner EF: AP-1: a double-edged sword in tumorigenesis. Nat Rev Cancer 2003;3:859-868.

5 Shaulian E, Karin M: AP-1 as a regulator of cell life and death. Nat Cell Biol 2002;4:E131-E136.

-6 Doble BW, Woodgett JR: GSK-3: tricks of the trade for a multi-tasking kinase. J Cell Sci 2003;116:11751186.

7 Wenemoser D, Lapan SW, Wilkinson AW, Bell GW, Reddien PW: A molecular wound response program associated with regeneration initiation in planarians. Genes Dev 2012;26:988-1002.

8 Imai KS, Hino K, Yagi K, Satoh N, Satou Y: Gene expression profiles of transcription factors and signaling molecules in the ascidian embryo: towards a comprehensive understanding of gene networks. Development 2004;131:4047-4058.

9 Kockel L, Homsy JG, Bohmann D: Drosophila AP-1: lessons from an invertebrate. Oncogene 2001;20:23472364.

10 Hess J, Angel P, Schorpp-Kistner M: AP-1 subunits: quarrel and harmony among siblings. J Cell Sci 2004;117:5965-5973.

11 Liu X, Guo CY, Ma XJ, Wu CF, Zhang Y, Sun MY, Pan YT, Yin HJ: Anti-inflammatory effects of Tanshinone IIA on atherosclerostic vessels of Oovariectomized ApoE-/- mice are mediated by estrogen receptor activation and through the ERK signaling pathway. Cell Physiol Biochem 2015;35:1744-1755. 


\section{Cellular Physiology Cell Physiol Biochem 2017;42:952-964 \begin{tabular}{l|l} 
DOI: 10.1159/000478678 & $\begin{array}{l}\text { O 2017 The Author(s). Published by S. Karger AG, Basel } \\
\text { www.karger.com/cpb }\end{array}$
\end{tabular} \\ Agron et al.: AP-1 Regulation by MAPKs in Cnidaria}

12 Yang J, Guo X, Yang J, Ding J, Li S, Yang R, Fan Z, Yang C: RP105 protects against apoptosis in ischemia/ reperfusion-induced myocardial damage in rats by suppressing TLR4-mediated signaling pathways. Cell Physiol Biochem 2015;36:2137-2148.

13 Putnam N, Srivastava M, Hellsten U, Dirks B, Chapman J, Salamov A, Terry A, Shapiro H, Lindquist E, Kapitonov V, Jurka J, Genikhovich G, Grigoriev I, Lucas S, Steele R, Finnerty J, Technau U, Martindale M, Rokhsar D: Sea anemone genome reveals ancestral eumetazoan gene repertoire and genomic organization. Science 2007;317:86 - 94.

14 Park E, Hwang D-S, Lee J-S, Song J-I, Seo T-K, Won Y-J: Estimation of divergence times in cnidarian evolution based on mitochondrial protein-coding genes and the fossil record. Mol Phylogenet Evol 2012;62:329-345.

-15 Hughes TP, Baird AH, Bellwood DR, Card M, Connolly SR, Folke C, Grosberg R, Hoegh-Guldberg O, Jackson JBC, Kleypas J, Lough JM, Marshall P, Nystrom M, Palumbi SR, Pandolfi JM, Rosen B, Roughgarden J: Climate change, human impacts, and the resilience of coral reefs. Science 2003;301:929-933.

16 Hoegh-Guldberg O, Mumby PJ, Hooten AJ, Steneck RS, Greenfield P, Gomez E, Harvell CD, Sale PF, Edwards AJ, Caldeira K, Knowlton N, Eakin CM, Iglesias-Prieto R, Muthiga N, Bradbury RH, Dubi A, Hatziolos ME: Coral reefs under rapid climate change and ocean acidification. Science 2007;318:1737-1742.

17 Mitchelmore CL, Ringwood AH, Weis VM: Differential accumulation of cadmium and changes in glutathione levels as a function of symbiotic state in the sea anemone Anthopleura elegantissima. J Exp Mar Biol Ecol 2003;284:71-85.

18 Sabdono A: Heavy metal levels and their potential toxic effect on coral Galaxea fascicularis from Java Sea, Indonesia. Res J Environ Sci 2009;3:96-102.

19 Brock JR, Bielmyer GK: Metal accumulation and sublethal effects in the sea anemone, Aiptasia pallida, after waterborne exposure to metal mixtures. Comp Biochem Physiol C Toxicol Pharmacol 2013;158:150-158.

20 Hand C, Uhlinger K: The unique, widely distributed, estuarine sea anemone, Nematostella vectensis; Stephenson: A review, new facts, and questions. Estuar Coasts 1994;17:501-508.

-21 Goldstone J: Environmental sensing and response genes in cnidaria: the chemical defensome in the sea anemone Nematostella vectensis. Cell Biol Toxicol 2008;24:483-502.

-22 Reitzel AM, Sullivan JC, Traylor-knowles N, Finnerty JR: Genomic survey of candidate stress-response genes in the estuarine anemone Nematostella vectensis. Biol Bull 2008;214:233-254.

23 Ambrosone A, Marchesano V, Mazzarella V, Tortiglione C: Nanotoxicology using the sea anemone Nematostella vectensis: from developmental toxicity to genotoxicology. Nanotoxicology 2014;8:508-520.

24 Harter VL, Mattews RA: Acute and chronic toxicity test methods for Nematostella vectensis Stephenson. Bull Environ Contam Toxicol 2005;74:830-836.

25 Elran R, Raam M, Kraus R, Brekhman V, Sher N, Plaschkes I, Chalifa-Caspi V, Lotan T: Early and late response of Nematostella vectensis transcriptome to heavy metals. Mol Ecol 2014;23:4722-4736.

26 DuBuc T, Traylor-Knowles N, Martindale M: Initiating a regenerative response, cellular and molecular features of wound healing in the cnidarian Nematostella vectensis. BMC Biol 2014;12:24.

27 Petersen HO, Höger SK, Looso M, Lengfeld T, Kuhn A, Warnken U, Nishimiya-Fujisawa C, Schnölzer M, Krüger M, Özbek S, Simakov O, Holstein TW: A comprehensive transcriptomic and proteomic analysis of Hydra head regeneration. Mol Biol Evol 2015:msv079.

-28 Wenger Y, Buzgariu W, Reiter S, Galliot B: Injury-induced immune responses in Hydra. Semin Immunol 2014;26:277-294.

29 Siboni N, Abrego D, Motti CA, Tebben J, Harder T: Gene expression patterns during the early stages of chemically induced larval metamorphosis and settlement of the coral Acropora millepora. PLoS ONE 2014;9:e91082.

-30 Rentzsch F, Fritzenwanker JH, Scholz CB, Technau U: FGF signalling controls formation of the apical sensory organ in the cnidarian Nematostella vectensis. Development 2008;135:1761-1769.

-31 Layden MJ, Johnston H, Amiel AR, Havrilak J, Steinworth B, Chock T, Röttinger E, Martindale MQ: MAPK signaling is necessary for neurogenesis in Nematostella vectensis. BMC Biol 2016;14:61.

-32 Marlow H, Matus DQ Martindale MQ: Ectopic activation of the canonical wnt signaling pathway affects ectodermal patterning along the primary axis during larval development in the anthozoan Nematostella vectensis. Dev Biol 2013;380:324-334.

-33 Trevino M, Stefanik DJ, Rodriguez R, Harmon S, Burton PM: Induction of canonical Wnt signaling by alsterpaullone is sufficient for oral tissue fate during regeneration and embryogenesis in Nematostella vectensis. Dev Dyn 2011;240:2673-2679. 


\section{Cellular Physiology Cell Physiol Biochem 2017;42:952-964 \begin{tabular}{l|l} 
DOI: 10.1159/000478678 & and Biochemistry \\
Published online: June 27, 2017 & $\begin{array}{l}\text { O 2017 The Author(s). Published by S. Karger AG, Basel } \\
\text { www.karger.com/cpb }\end{array}$
\end{tabular} \\ Agron et al.: AP-1 Regulation by MAPKs in Cnidaria}

34 Katoh K, Standley DM: MAFFT Multiple Sequence Alignment Software Version 7: Improvements in Performance and Usability. Mol Biol Evol 2013;30:772-780.

-35 Xia X: DAMBE5: A Comprehensive Software Package for Data Analysis in Molecular Biology and Evolution. Mol Biol Evol 2013;30:1720-1728.

36 Huelsenbeck JP, Ronquist F: MRBAYES: Bayesian inference of phylogenetic trees. Bioinformatics 2001;17:754-755.

-37 Rambaut A, Suchard M, Xie D, Drummond A: Tracer v1. 6, 2014,

-38 Holland PM, Cooper JA: Protein modification: docking sites for kinases. Curr Biol 1999;9:R329-R331.

-39 Sutherland JA, Cook A, Bannister AJ, Kouzarides T: Conserved motifs in Fos and Jun define a new class of activation domain. Genes Dev 1992;6:1810-1819.

40 Grimes CA, Jope RS: The multifaceted roles of glycogen synthase kinase $3 \beta$ in cellular signaling. Prog Neurobiol 2001;65:391-426.

-41 Cuadrado A, Nebreda Angel R: Mechanisms and functions of p38 MAPK signalling. Biochem J 2010;429:403-417.

42 Kültz D: Phylogenetic and functional classification of mitogen- and stress-activated protein kinases. J Mol Evol 1998;46:571-588.

43 Johnson GL, Nakamura K: The c-jun kinase/stress-activated pathway: regulation, function and role in human disease. Biochim Biophys Acta Mol Cell Res 2007;1773:1341-1348.

-44 Young PR, McLaughlin MM, Kumar S, Kassis S, Doyle ML, McNulty D, Gallagher TF, Fisher S, McDonnell PC, Carr SA: Pyridinyl imidazole inhibitors of p38 mitogen-activated protein kinase bind in the ATP site. J Biol Chem 1997;272:12116-12121.

-45 Nithianandarajah-Jones GN, Wilm B, Goldring CEP, Müller J, Cross MJ: ERK5: Structure, regulation and function. Cell Signal 2012;24:2187-2196.

-46 Terasawa K, Okazaki K, Nishida E: Regulation of c-Fos and Fra-1 by the MEK5-ERK5 pathway. Genes Cells 2003;8:263-273.

-47 Tanoue T, Adachi M, Moriguchi T, Nishida E: A conserved docking motif in MAP kinases common to substrates, activators and regulators. Nat Cell Biol 2000;2:110-116.

$>48$ Yang Q Deng X, Lu B, Cameron M, Fearns C, Patricelli MP, Yates JR, Gray NS, Lee J-D: Pharmacological inhibition of BMK1 suppresses tumor growth through PML. Cancer Cell 2010;18:258-267.

-49 Zeller E, Hammer K, Kirschnick M, Braeuning A: Mechanisms of RAS/ $\beta$-catenin interactions. Arch Toxicol 2013;87:611-632.

-50 Dajani R, Fraser E, Roe SM, Young N, Good V, Dale TC, Pearl LH: Crystal structure of glycogen synthase kinase $3 \beta$ : structural basis for phosphate-primed substrate specificity and autoinhibition. Cell 2001;105:721-732.

51 Jindrich K, Degnan BM: The diversification of the basic leucine zipper family in eukaryotes correlates with the evolution of multicellularity. BMC Evol Biol 2016;16:1.

52 Reinke AW, Baek J, Ashenberg 0, Keating AE: Networks of bZIP protein-protein interactions diversified over a billion years of evolution. Science 2013;340:730-734.

-53 Amoutzias G, Veron A, Weiner J, Robinson-Rechavi M, Bornberg-Bauer E, Oliver S, Robertson D: One billion years of bZIP transcription factor evolution: conservation and change in dimerization and DNA-binding site specificity. Mol Biol Evol 2007;24:827-835.

54 van de Water JA, Leggat W, Bourne DG, van Oppen MJ, Willis BL, Ainsworth TD: Elevated seawater temperatures have a limited impact on the coral immune response following physical damage. Hydrobiologia 2015;759:201-214.

55 Matsuoka M, Igisu H: Effects of heavy metals on mitogen-activated protein kinase pathways. Environ Health Prevent Med 2002;6:210-217.

56 Cohen P, Frame S: The renaissance of GSK3. Nature Reviews Molecular Cell Biology 2001;2:769-776.

57 Tullai JW, Tacheva S, Owens LJ, Graham JR, Cooper GM: AP-1 is a component of the transcriptional network regulated by GSK-3 in quiescent cells. PLoS ONE 2011;6:e20150.

58 Liang M-H, Chuang D-M: Differential roles of glycogen synthase kinase-3 isoforms in the regulation of transcriptional activation. J Biol Chem 2006;281:30479-30484. 